\title{
Continuous Rocuronium Administration Method Based on Pharmacokinetic/ Pharmacodynamics Model during Propofol, Sevoflurane, and Desflurane Anesthesia
}

\author{
Takahiro Moriyama*, Yoshihiko Irie, Keika Mukaihara, Kanako Ishizuka, Akira Matsunaga, \\ Yuichi Kanmura \\ Department of Anesthesiology and Intensive Care, Kagoshima University Hospital, Kagoshima, Japan \\ Email: *takmor@m3.kufm.kagoshima-u.ac.jp
}

Received 13 April 2016; accepted 28 May 2016; published 31 May 2016

Copyright @ 2016 by authors and Scientific Research Publishing Inc.

This work is licensed under the Creative Commons Attribution International License (CC BY).

http://creativecommons.org/licenses/by/4.0/

(c) (i) Open Access

\begin{abstract}
Purpose: Although rocuronium bromide (Rb) is suitable for continuous administration use, determination of optimal continuous doses is difficult due to individual differences. This study examines the efficacy of a continuous $\mathrm{Rb}$ administration method based on effect-site concentrations calculated by a pharmacokinetic/pharmacodynamics model during propofol, sevoflurane, and desflurane anesthesia. Methods: The 36 enrolled patients were equally divided into three groups (P; propofol, S; sevoflurane, and D; desflurane groups). After induction and administration of Rb $0.6 \mathrm{mg} / \mathrm{kg}$, we calculated the simulated effect-site concentration at the point which the first twitch (\%T1) recovered to $>0 \%$ and defined this as the $\mathrm{Rb}$ recovery concentration (Rbr.c.) level appropriate for continuous rocuronium administration. The continuous administration doses of $\mathbf{R b}$ were adjusted to maintain Rbr.c. during surgery. The Rbr.c. and the recovery time at \%T1 $>25 \%$ were recorded for each type of anesthesia. Results: Rbr.c. $(\mu \mathrm{g} / \mathrm{mL})$ for the $P, S$, and $D$ groups were $1.54 \pm 0.2,1.24 \pm 0.2$, and $1.09 \pm 0.2$, respectively. Continuous administration doses $(\mu \mathrm{g} / \mathrm{kg} / \mathrm{min})$ in the $P, S$, and $D$ group were $6.7 \pm 0.9,5.2 \pm 1.0$, and $4.5 \pm 0.8$, respectively. Rbr.c. and continuous doses in the $S$ and $D$ groups were lower than the $P$ group. Neuromuscular relaxations during surgery in the $S$ and $D$ groups were more strongly maintained than for the $P$ group. There was also a significantly prolonged recovery duration for the \%T1 $>25 \%$ in the D versus the other two groups $(P<0.05)$. Conclusion: Results showed that our continuous administration method was effective for maintaining sufficient muscle relaxation without excessively prolonged recovery effects for both sevoflurane and desflurane as well as propofol anesthesia.
\end{abstract}

*Corresponding author.

How to cite this paper: Moriyama, T., Irie, Y., Mukaihara, K., Ishizuka, K., Matsunaga, A. and Kanmura, Y. (2016) Continuous Rocuronium Administration Method Based on Pharmacokinetic/Pharmacodynamics Model during Propofol, Sevoflurane, and Desflurane Anesthesia. Open Journal of Anesthesiology, 6, 77-84. http://dx.doi.org/10.4236/ojanes.2016.65013 


\section{Keywords}

\section{Rocuronium, Continuous Administration, Propofol, Sevoflurane, Desflurane}

\section{Introduction}

Neuromuscular blocking agents are commonly administered intermittently. When given as a bolus infusion, however, large differences in the blood concentrations are noted between the before and after infusion levels. Thus, this can cause an undesired overdose or insufficient muscle relaxation during general anesthesia. Since rocuronium bromide $(\mathrm{Rb})$ has a rapid onset, intermediates duration of action, and exhibits almost no accumulation effect, $\mathrm{Rb}$ is considered to be suitable for use by continuous infusion [1] [2]. As compared to the use of $\mathrm{Rb}$ bolus infusions, continuous administration of $\mathrm{Rb}$ is able to stably maintain both the blood and effect-site concentrations, and provides sufficient muscle relaxation effects during surgery without any observed body movements or bucking. However, there are large interindividual differences in the sensitivity to $\mathrm{Rb}$ due to several factors, such as gender, age, body weight, skeletal muscle mass, and sensitivity to neuromuscular effects [3]-[7]. Therefore, it is essential to be able to determine the optimal rates of continuous administration of Rb in accordance with the individual patient. We previously reported an effective continuous rocuronium administration method that was based on the simulated effect-site concentrations obtained from a pharmacokinetic/pharmacodynamics model during propofol anesthesia [8]. Our results showed that the simulated rocuronium concentration at the time of recovery to a first twitch (\%T1) $>0 \%$ after the initial bolus administration of rocuronium was a good indicator of the optimal effect-site concentrations that would occur during continuous rocuronium administration under propofol anesthesia. Based on the previous result, we hypothesized that this continuous administration method was also effective under inhaled anesthesia, because continuous administration doses of $\mathrm{Rb}$ in our protocol were decided in accordance to each patient under each anesthesia. In the present study, we further compared the efficacy of this method when using propofol, sevoflurane, and desflurane anesthesia. The specific aim of the present study was to clarify whether our method when using sevoflurane and desflurane anesthesia was as effective as that observed for propofol.

\section{Methods}

The present study was approved by the Ethics Committee of Kagoshima University Hospital and registered with the UMIN Clinical Trials Registry (UMIN 000012313) on November 18, 2013. It was conducted in accordance with the principles of the Declaration of Helsinki, and prior written informed consent was obtained from each patient.

This study enrolled 36 adult patients under 65 years of age with an American Society of Anesthesiologists physical class status of 1 to 2 who were undergoing elective general surgery. Patients were equally and randomly divided into three groups (P; propofol, S; sevoflurane, and D; desflurane groups). Patients with renal, hepatic, or neuromuscular diseases were excluded. Patients receiving medications known to interfere with neuromuscular blocking agents, such as antibiotics and anticonvulsants, as well as obese patients (BMI $>30 \mathrm{~kg} / \mathrm{m}^{2}$ ), were also excluded. All patients fasted overnight and were not premedicated. On arrival in the operating room, patients were monitored with electrocardiography, non-invasive blood pressure, pulse oximetry, and bispectral index (BIS; Aspect Medical Systems, Norwood, MA, USA). Under local anesthesia, an intravenous catheter was inserted into a forearm vein and a cannula was inserted into the radial artery. Neuromuscular monitoring of the other arm was performed using a train-of-four watch (TOF Watch SX; Organon, Osaka, Japan). Stabilization and calibration of the monitoring device was performed according to good clinical research practices in pharmacodynamic studies of neuromuscular blocking agents [9]. After the acceleration transducer was placed in the hand adaptor, the fingers were fixed to an arm board.

Anesthesia was induced with propofol and remifentanil. After induction of anesthesia and stabilization of the muscle response to ulnar nerve stimulation, the TOF monitor was calibrated to obtain maximal nerve stimulation.

After induction, rocuronium bromide at a dose of $0.6 \mathrm{mg} / \mathrm{kg}$ of total body weight (TBW) was administered intravenously. Nerve stimulation using the single stimulation mode at $1 \mathrm{~Hz}$ was then conducted every 15 seconds. 
When the \%T1 decreased to $0 \%$, which indicates a full neuromuscular block, tracheal intubation was performed. All patients were mechanically ventilated during their surgery. In the P group, the propofol infusion was adjusted based on the TBW using a target-controlled infusion pump (TE-371TCI; Terumo Corporation, Tokyo, Japan). During all experimental procedures, propofol was started to infuse with a target concentration of $3 \mu \mathrm{g} / \mathrm{ml}$. Both sevoflurane and desflurane were started to inhale at 0.6 minimum alveolar concentration (MAC) (end-tidal concentrations were $1 \%$ for sevoflurane and $3.6 \%$ for desflurane) in the S and D groups. Infusion rate of propofol and inhaled concentrations of sevoflurane and desflurane were adjusted in order to maintain the BIS value within a range of 40 - 60. In all cases, the remifentanil infusion was adjusted depending on surgical invasiveness and based on the ideal body weight (IBW), which was calculated as height $(\mathrm{m})^{2} \times 22$, within the range of 0.1 $0.5 \mu \mathrm{g} / \mathrm{kg} / \mathrm{min}$. The doses of propofol and rocuronium were adjusted according to the TBW. During the studies, the core temperature was maintained above $36.0^{\circ} \mathrm{C}$ and the palm temperature above $32.0^{\circ} \mathrm{C}$ by using a Bair Hugger $^{\circledR}$ forced-air warming device (Arizant Healthcare, Inc., Eden Prairie, MN, USA).

When the \% 1 increased to more than $3 \%$ in successive measurements after the initial administration of rocuronium, we defined the effect-site concentration of Rb determined by the Wierda pharmacokinetic-pharmacodynamic model [10] as the rocuronium bromide recovery concentration (Rbr.c.). At this point, we then started a $7 \mu \mathrm{g} / \mathrm{kgTBW} / \mathrm{min}$ continuous administration of $\mathrm{Rb}$. In order to maintain the Rbr.c. during the surgery, the administration rate was adjusted by changing the infusion rate of $\mathrm{Rb}$ in increments or decrements of $1 \mu \mathrm{g} / \mathrm{kg} / \mathrm{min}$ every 10 minutes. When adjusting the rocuronium infusion rate, the anesthesiologist did not refer to the state of the neuromuscular relaxation that was shown on the neuromuscular monitor. If a patient exhibited a \%T1 increase of more than $20 \%$ during the continuous $\mathrm{Rb}$ administration, the experimental protocol dictated that the experiment was to be immediately discontinued. Just prior to the end of the surgery, Rb administration was discontinued, and the time until recovery of the \%T1 to over $25 \%$ was measured along with the effect-site concentrations at this particular point, and we administered $0.2 \mathrm{mg} / \mathrm{kg}$ sugammadex at the recovery point of \%T1 > $25 \%$.

\section{Statistical Analysis}

All results are shown as the mean and the standard deviation (SD). We analyzed the differences among the three groups using one-way analysis of variance (ANOVA), and post-hoc test was conducted using Bonferroni method. $P$-values $<0.05$ were considered statistically significant. Statistical analysis was performed using Graphic Prism version 5.0 software (GraphPad Software, San Diego, CA, USA).

\section{Results}

Table 1 presents the characteristics of the 36 patients. The Rbr.c. $(\mu \mathrm{g} / \mathrm{ml})$ for the $\mathrm{P}, \mathrm{S}$, and D groups were $1.54 \pm$ $0.2,1.24 \pm 0.2$, and $1.09 \pm 0.2$, respectively. The Rbr.c. in the P group was significantly higher than that observed in both the $\mathrm{S}$ and D groups. $(P<0.05$; Figure 1$)$. Recovery concentrations $(\mu \mathrm{g} / \mathrm{mL})$ at the time that the \%T1 returned to over $25 \%$ were significantly higher in the $\mathrm{P}$ versus the $\mathrm{S}$ and $\mathrm{D}$ groups $(P<0.05$; Figure 2(a)). Recovery durations (min) required for the $\% \mathrm{~T} 1$ to return to over $25 \%$ in the $\mathrm{P}, \mathrm{S}$, and D groups were $15.7 \pm$

\section{Table 1. Patient demographic data.}

\begin{tabular}{cccc}
\hline & $\mathrm{P}(\mathrm{n}=12)$ & $\mathrm{S}(\mathrm{n}=12)$ & $\mathrm{D}(\mathrm{n}=12)$ \\
\hline Male: Female & $6: 6$ & $6: 6$ & $6: 6$ \\
Age (year) & $49.6 \pm 12.5$ & $46.2 \pm 14.0$ & $44.3 \pm 13.0$ \\
Height (cm) & $163.0 \pm 5.7$ & $160.4 \pm 8.0$ & $160.2 \pm 6.7$ \\
Weight (kg) & $61.7 \pm 9.7$ & $57.5 \pm 9.1$ & $58.3 \pm 10.9$ \\
Body mass index (kg·m $\left.{ }^{-2}\right)$ & $23.5 \pm 3.0$ & $22.2 \pm 2.5$ & $22.8 \pm 3.2$ \\
Operating time (min) & $222.2 \pm 78.3$ & $233.4 \pm 90.4$ & $243.1 \pm 60.0$ \\
Anesthesia time (min) & $306.4 \pm 85.4$ & $334.7 \pm 88.8$ & $351.3 \pm 47.2$ \\
\hline
\end{tabular}

Data are expressed as mean \pm standard deviation range and ratio of patients. There were no significant differences for the background among the three groups. P; propofol. S; sevoflurane. D; desflurane. 
7.0, $19.6 \pm 7.6$, and $28.2 \pm 6.3$, respectively, with the recovery duration significantly prolonged in the D versus the other two groups $(P<0.05$; Figure 2(b)). Continuous administration doses $(\mu \mathrm{g} / \mathrm{kg} / \mathrm{min})$ in the $\mathrm{P}, \mathrm{S}$, and $\mathrm{D}$ groups were $6.7 \pm 0.9,5.2 \pm 1.0$, and $4.5 \pm 0.8$, respectively. The level of the continuous doses in the $\mathrm{S}$ and $\mathrm{D}$ groups were significantly lower than that observed in the P group (Figure 3). Figure 4 shows the ratio for the \% 1 values during the continuous administration of $\mathrm{Rb}$ (calculated as a percentage). The muscle relaxation effects for both sevoflurane and desflurane anesthesia were stronger than that observed when under propofol anesthesia.

\section{Discussion}

In the present study, the result showed that neuromuscular relaxations could be maintained without excessively prolonged effects under both sevoflurane and desflurane anesthesia, as well as propofol anesthesia. More sufficient and stable neuromuscular relaxation under inhaled anesthesia could be taken, therefore it was considered that recovery time for achieving \% $11>25 \%$ was required longer that of the propofol anesthesia.

As compared with the propofol anesthesia, the Rbr.c., which was defined as the concentration of $\mathrm{Rb}$ at the point where the recovery of the \%T1 was $>0 \%$ after the bolus administration, was significantly lower for both the sevoflurane and desflurane anesthesia in the current study. Previous researchers have reported that the effects of

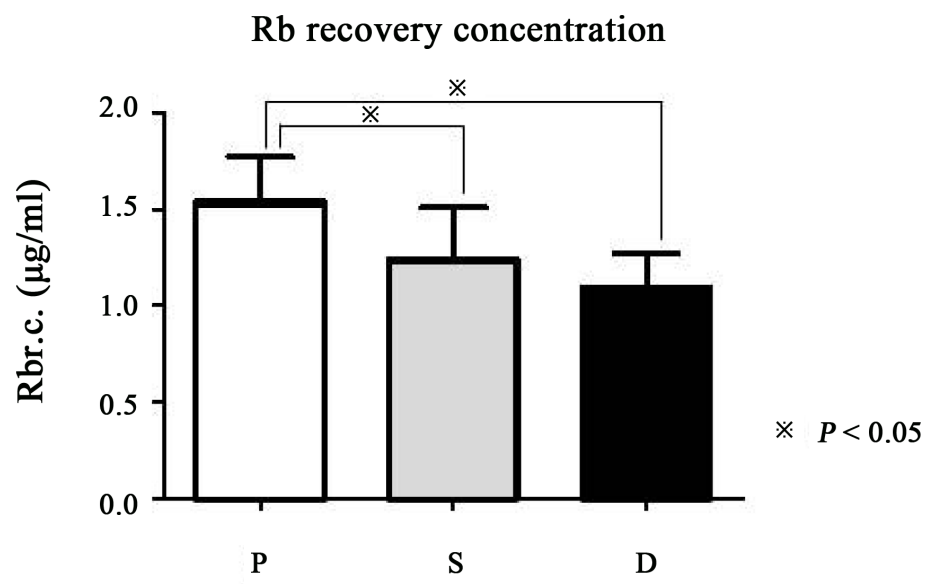

Figure 1. Rbr.c. $(\mu \mathrm{g} / \mathrm{mL})$ of the $\mathrm{P}, \mathrm{S}$, and D groups were $1.54 \pm 0.2,1.24 \pm 0.2$, and $1.09 \pm 0.2$, respectively. Rbr.c. was significantly higher in the P group compared to the in the $\mathrm{S}$ and $\mathrm{D}$ groups $(P<0.05)$. $\mathrm{P}$; propofol. S; sevoflurane. D; desflurane.
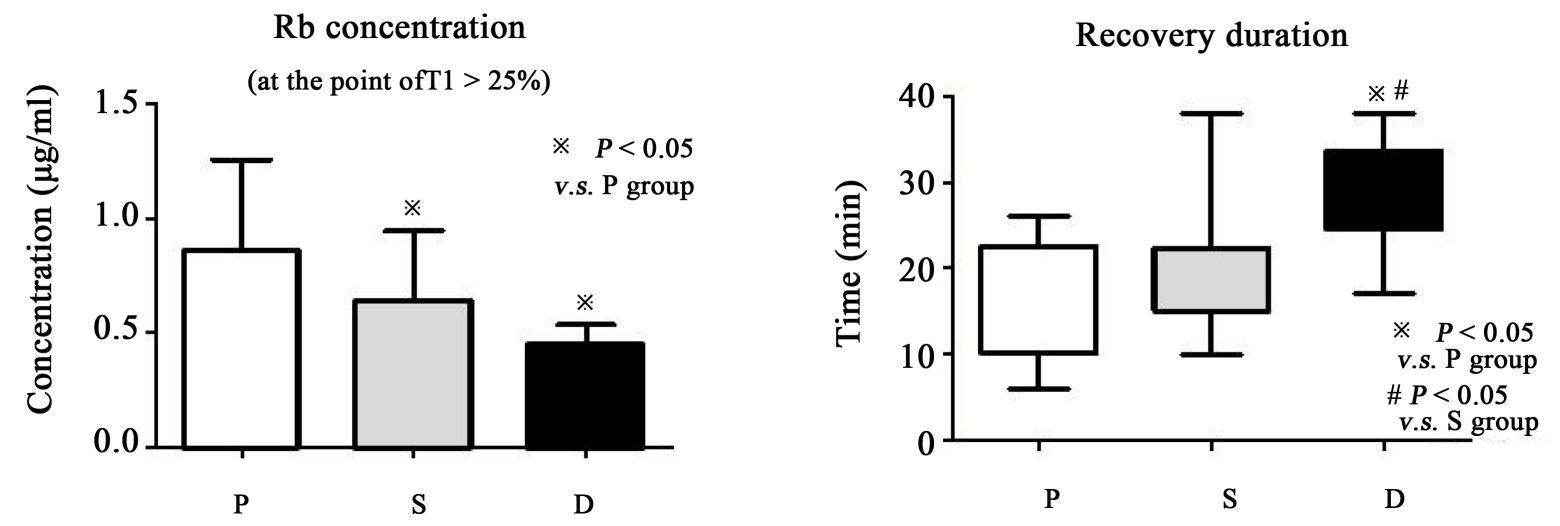

Figure 2. (a) Simulated effect-site concentrations of the $\mathrm{Rb}(\mu \mathrm{g} / \mathrm{mL})$ at the time of recovery of the \%T1 to over $25 \%$ in the P, $\mathrm{S}$, and D groups were $0.86 \pm 0.4,0.64 \pm 0.3$, and $0.45 \pm 0.1$, respectively. There were significantly lower Rb concentrations in the $\mathrm{S}$ and $\mathrm{D}$ groups at these points, as compared to the $\mathrm{P}$ group $(P<0.05)$. P; propofol. S; sevoflurane. D; desflurane. (b) Recovery durations (min) until the \%T1 recover to over $25 \%$ in the $\mathrm{P}, \mathrm{S}$, and D groups were $15.7 \pm 7.0$ ), 19.6 \pm 7.6 , and $28.2 \pm 6.3$, respectively. The recovery duration in the $\mathrm{D}$ group was significantly prolonged as compared to the $\mathrm{P}$ and $\mathrm{S}$ groups $(P<0.05)$. P; propofol. S; sevoflurane. D; desflurane. 
$\mathrm{Rb}$ administration dose

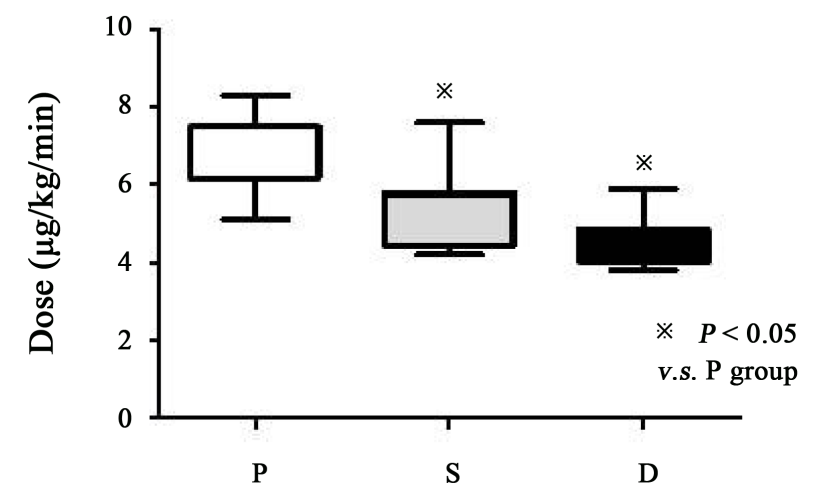

Figure 3. Continuous administration doses $(\mu \mathrm{g} / \mathrm{kg} / \mathrm{min})$ in the $\mathrm{P}$, $\mathrm{S}$, and D group were $6.7 \pm 0.9,5.2 \pm 1.0$, and $4.5 \pm 0.8$, respectively. The continuous doses in the $\mathrm{S}$ and $\mathrm{D}$ groups were significantly lower than the dose in the P group. P; propofol. S; sevoflurane. D; desflurane.

\section{T1 values}

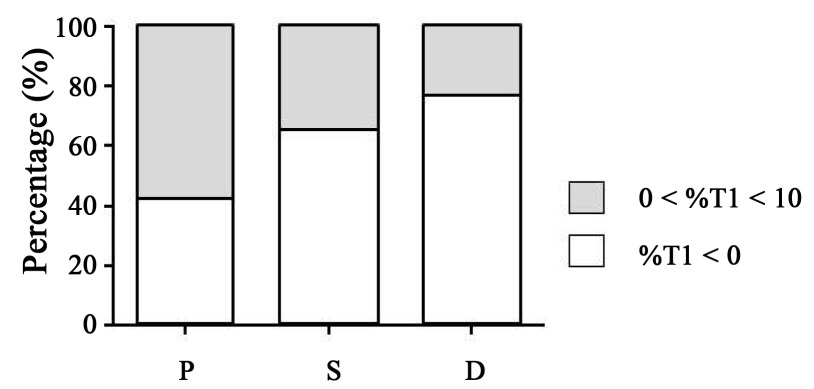

Figure 4. The ratios for the \%T1 values after calculation as a percentage. The percentages for the \%T1 $<0$ and the $0<\% \mathrm{~T} 1<$ 10 values are shown below: P group; \%T1 $<0: 0<\% \mathrm{~T} 1<10=$ 43.1\%:56.9\%. S group; \%T1 < 0:0 < \% $1<10=65.4 \%: 34.6 \%$. D group; \%T1 < 0:0 < \% $1<10=75.3 \%: 24.7 \%$. P; propofol. S; sevoflurane. D; desflurane.

neuromuscular blocking agents are prolonged when using inhaled anesthesia such as sevoflurane, isoflurane and desflurane [11]-[13]. While desflurane by itself exhibits few neuromuscular blocking effects when used at clinical concentrations [14]. It has been shown to enhance the effects of neuromuscular blocking agents to the same degree as seen for sevoflurane [11]. In contrast, propofol has neither neuromuscular blocking nor enhancing effects. Therefore, many other studies have shown that the potency of Rb was increased by $25 \%-40 \%$ during inhalation anesthesia with agents other than propofol [12] [13]. These previous studies which were conducted with an inhaled anesthesia at MAC of over 1, showed that the effects of the neuromuscular blocking were dependent upon both the time and the concentration of the inhaled anesthetics. In our current study, we adopted 0.6 MAC for the maintenance concentrations of sevoflurane and desflurane, as we were also administering remifentanil, which reduces the needed concentrations of inhaled anesthetics.

By predeterming and adjusting an adequate continuous administration $\mathrm{Rb}$ doses in accordance to each anesthetic agent, it should be possible to maintain an efficient neuromuscular relaxation during surgery and to avoid prolonged recovery times. For example, Bock et al. reported that as compared to propofol, the doses for the continuous administration of $\mathrm{Rb}$ that were needed to maintain the same degree of neuromuscular relaxation were smaller for sevoflurane and desflurane [12]. In addition, even though the doses were smaller, there were no significant differences among three anesthetic drugs for the recovery time from the neuromuscular blocking condi- 
tions. Another study reported that the continuous administration rates of Rb required to keep the \%T1 between $3 \%-10 \%$ were lower for sevoflurane versus propofol anesthesia [15]. In the present study, we also found that the required continuous administration doses were lower for the inhaled versus the propofol anesthesia. However, the neuromuscular relaxant effects were stronger and there was a longer time to recovery for $\% \mathrm{~T} 1>25 \%$ for the inhaled anesthetic agents. With regard to our current results, it should be noted that the surgical times for our study were longer as compared to the previous studies, and that the inhaled anesthesia, especially sevoflurane, was able to increase and enhance the neuromuscular relaxation effects [16]. Even though these differences could have an influence to our results, our study found that the continuous administration doses of Rb were smaller than that for the inhaled anesthesia cases where there were deeper neuromuscular relaxation conditions and significantly prolonged recovery durations from the effects after stopping the continuous administration of Rb.

In the present study, the recovery times for achieving \%T1 > 25\% were $15.7 \pm 7.0,19.6 \pm 7.6$ and $28.2 \pm 6.3$ minutes for the P, S and D groups, respectively. Although the longest recovery time needed for a desflurane anesthesia case was $38 \mathrm{~min}$, we did not observe any other cases with excessively prolonged recovery duration. We found the data for a recovery to $\% \mathrm{~T} 1>25 \%$ to be both sufficient and within an acceptable range for clinical use. Thus, this information should be of great benefit when evaluating the conditions of the patients and determining when it is both appropriate and safe to stop the continuous administration of Rb. Moreover, the administration of sugammadex can be used to completely antagonize any neuromuscular blocking effects induced by Rb, as the reversal effects of sugammadex are equally effective for both sevoflurane and propofol [17]. Indeed, in our study, we confirmed that the neuromuscular relaxation effects were completely and rapidly reversed to \%T1 > $100 \%$ in all cases when we administered sugammadex at the recovery point of $\% \mathrm{~T} 1>25 \%$.

When using continuous administration of $\mathrm{Rb}$, the blood concentrations are more stable, which helps to keep the patients more stable and improve the surgical conditions. Previous experimental findings have demonstrated the advantages of using Rb, which exhibits no accumulation and has very few potent metabolites [18]. While several continuous administration methods, including target control infusion methods, have been examined and proposed for routine use [19], there have been many difficulties encountered clinically. These problems have been speculated to be primarily caused by the large individual variations that are seen in the patients. Thus, we examined the efficacy of a new method of continuous administration of Rb that uses the Rbr.c. as the indicator for determining the optimal level of anesthesia.

Neuromuscular relaxation effects were found to be stronger when using inhaled anesthesia, especially desflurane. Although we defined the Rbr.c. values after the first bolus administration of Rb, there is a possibility that the inhaled anesthesia could have had an enhancing effect on the neuromuscular relaxation during the continuous administration of $\mathrm{Rb}$. The gold standard for neuromuscular monitoring is the use of the TOF Watch to monitor the contraction of the adductor pollicis muscle [9]. However, even if there is complete paralysis of the adductor pollicis muscle, other muscles such as the diaphragm and the abdominal muscles might be able to partially recover from the neuromuscular blockade [20]. Since this could lead to moving or bucking of the patients and lead to an inadequate surgical field during the surgery, deeper neuromuscular relaxation effects are occasionally needed. Our current study showed that the \%T1 values could be maintained in all cases at $<10 \%$ during a continuous Rb administration. In fact, in nearly half of the cases under propofol anesthesia and throughout the majority of the duration while under the inhaled anesthesia, the $\% \mathrm{~T} 1$ values were $0 \%$. In addition, there were no harmful events such as moving and bucking of patients or any claims of an inadequate field by surgeons during any of the surgeries. Therefore, we believe that our continuous administration method is able to maintain sufficient neuromuscular relaxation conditions under all general anesthesia procedures, especially during inhaled anesthesia.

One of the limitations of our current study was that we could not show any correlation between the duration of a continuous $\mathrm{Rb}$ administration and the time to achieve a recovery of \% $1>25 \%$ (data not shown). Even so, it should be noted that the inhaled anesthesia was able to induce enhancing neuromuscular relaxant effects in a time-dependent manner. Moreover, as compared to propofol and to sevoflurane, desflurane exhibited stronger neuromuscular relaxant effects and required a longer time to achieve a recovery of \%T1 $>25 \%$. Previous studies have reported that even though the results were not significant, desflurane did tend to enhance the neuromuscular blocking to a greater degree than sevoflurane [10] [11]. Comparisons between desflurane and sevoflurane regarding which concentrations of the inhaled anesthesia are most effective and the associated recovery times will need to be examined in further studies. 


\section{Conclusion}

The simulated effective concentration of $\mathrm{Rb}$ at the point where $\% \mathrm{~T} 1$ recovers to $>0 \%$ can be used as an indicator to define the optimal continuous administration rates of Rb. This continuous administration method of Rb is proved to be effective in maintaining sufficient and stable muscle relaxation without excessively prolonging the effects under sevoflurane and desflurane, as well as propofol anesthesia. As compared to propofol, inhaled anesthetics exhibit enhanced neuromuscular blocking effects, with the enhanced effects perhaps stronger under desflurane versus sevoflurane anesthesia.

\section{Conflict of Interest Statement}

None of authors or institute has received any financial or material support from the manufacture.

\section{Funding}

This study was supported in part by the university’s clinical research budget.

\section{References}

[1] Shanks, C.A., Fragen, R.J. and Ling, D. (1993) Continuous Intravenous Infusion of Rocuronium (ORG 9426) in Patients Receiving Balanced, Enflurane, or Isoflurane Anesthesia. Anesthesiology, 78, 649-651. http://dx.doi.org/10.1097/00000542-199304000-00006

[2] McCoy, E.P., Mirakhur, R.K., Maddineni, V.R., Loan, P.B. and Connolly, F. (1994) Administration of Rocuronium (Org 9426) by Continuous Infusion and Its Reversibility with Anticholinesterases. Anaesthesia, 49, 940-945. http://dx.doi.org/10.1111/j.1365-2044.1994.tb04308.x

[3] Xue, F.S., Tong, S.Y., Liao, X., Liu, J.H., An, G. and Luo, L.K. (1997) Dose-Response and Time Course of Effect of Rocuronium in Male and Female Anesthetized Patients. Anesthesia \& Analgesia, 85, 667-671.

[4] Bevan, D.R., Fiset, P., Balendran, P., Law-Min, J.C., Ratcliffe, A. and Donati, F. (1993) Pharmacodynamic Behavior of Rocuronium in the Elderly. Canadian Journal of Anesthesia, 40, 127-132. http://dx.doi.org/10.1007/BF03011309

[5] Leykin, Y., Pellis, T., Lucca, M., Lomangino, G., Marzano, B. and Gullo, A. (2004) The Pharmacodynamics Effects of Rocuronium When Dosed According to Real Body Weight or Ideal Body Weight in Morbidly Obese Patients. Anesthesia \& Analgesia, 99, 1086-1089. http://dx.doi.org/10.1213/01.ANE.0000120081.99080.C2

[6] Meyhoff, C.S., Lund, J., Jenstrup, M.T., Claudis, C., Sorensen, A.M., Viby-Mogensen, J. and Ramussen, L.S. (2009) Should Dosing of Rocuronium in Obese Patients Be Based on Ideal or Corrected Body Weight? Anesthesia \& Analgesia, 109, 787-792. http://dx.doi.org/10.1213/ane.0b013e3181b0826a

[7] Dahaba, A.A., Perelman, S.I., Moskowitz, D.M., Bennett, H.L., Shander, A., Xiano, Z., Huang, L., An, G., Bornemann, H., Wilfinger, G., Hager, B., Rehak, P.H., List, W.F. and Metzler, H. (2006) Geographic Regional Differences in Rocuronium Bromide Dose-Response Relation and Time Course of Action: An Overlooked Factor in Determining Recommended Dosage. Anesthesiology, 10, 950-953. http://dx.doi.org/10.1097/00000542-200605000-00010

[8] Moriyama, T., Matsunaga, A., Nagata, O., Enohata, K., Kamikawaji, T., Uchino, E. and Kanmura, Y. (2015) Effective Method of Continuous Rocuronium Administration Administration Based on Effect-Site Concentrations Using a Pharmacokinetic/Pharmacodynamics Model during Propofol-Remifentanil Anesthesia. Journal of Anesthesia, 29, 593599. http://dx.doi.org/10.1007/s00540-015-1991-2

[9] Fuchs-Buder, T., Claudis, C., Skovgaard, L.T., Eriksson, L.I., Mirakhur, R.K. and Viby-Monngensen, J. (2007) Good Clinical Research Practice in Pharmacodynamics Studies of Neuromuscular Blocking Agents II: The Stockholm Revision. Acta Anaesthesiologica Scandinavica, 51, 789-808. http://dx.doi.org/10.1111/j.1399-6576.2007.01352.X

[10] Wierda, J.M., Kleef, U.W., Lambalk, L.M., Kloppenburg, W.D. and Agoston, S. (1991) The Pharmacodynamics and Pharmacokinetics of Org 9426, a New Non-Depolarizing Neuromuscular Blocking Agent, in Patients Anaesthetized with Nitrous Oxide, Halothane and Fentanyl. Canadian Journal of Anesthesia, 38, 430-435. http://dx.doi.org/10.1007/BF03007578

[11] Wulf, H., Ledowski, T., Linstedt, U., Proppe, D. and Sitzlack, D. (1998) Neuromuscular Blocking Effects of Rocuronium during Desflurane, Isoflurane, and Sevoflurane Anaesthesia. Canadian Journal of Anesthesia, 45, 526-532. http://dx.doi.org/10.1007/BF03012702

[12] Bock, M., Klippel, K., Nitsche, B., Bach, A., Martin, E. and Motsch, J. (2000) Rocuronium Potency and Recovery Characteristics during Steady-State Desflurane, Sevoflurane, Isoflurane or Propofol Anaesthesia. British Journal of Anaesthesia, 84, 43-47. http://dx.doi.org/10.1093/oxfordjournals.bja.a013380

[13] Lowry, D.W., Mirakhur, R.K., McCarthy, G.J., Carroll, M.T. and McCourt, K.C. (1998) Neuromuscular Effects of 
Rocuronium during Sevoflurane, Isoflurane, and Intravenous Anesthesia. Anesthesia \& Analgesia, 87, 936-940.

[14] Caldwell, J.E., Laster, M.J., Magorian, T., Heier, T., Yasuda, N., Lynam, D.P., Eger, E.I. and Weiskopf, R.B. (1991) The Neuromuscular Effects of Desflurane, Alone and Combined with Pancuronium or Succinylcholine in Humans. Anesthesiology, 74, 412-418. http://dx.doi.org/10.1097/00000542-199103000-00004

[15] Takagi, S., Ozaki, M., Iwasaki, H., Hatano, Y. and Takeda, J. (2006) Effects of Sevoflurane and Propofol on Neuromuscular Blocking Action of Org 9426 (Rocuronium Bromide) Infused Continuously in Japanese Patients. Masui, 55, 963-970.

[16] Ahmed, A.A., Kumagai, M., Otake, T., Kurata, Y. and Amaki, Y. (1999) Sevoflurane Exposure Time and the Neuromuscular Blocking Effects of Vecuronium. Canadian Journal of Anesthesia, 46, 429-432. http://dx.doi.org/10.1007/BF03012941

[17] Vanacker, B.F., Vermeyen, K.M., Struys, M.M., Rietbergen, H., Vandermeersch, E., Saldien, V., Kalmar, A.F. and Prins, M.E. (2007) Reversal of Rocuronium-Induced Neuromuscular Block with the Novel Drug Sugammadex Is Equally Effective under Maintenance Anesthesia with Propofol or Sevoflurane. Anesthesia \& Analgesia, 104, 563-568. http://dx.doi.org/10.1213/01.ane.0000231829.29177.8e

[18] Wierda, J.M., Kleef, U.W., Lambalk, L.M., Kloppenburg, W.D. and Agoston, S. (1991) The Pharmacodynamics and Pharmacokinetics of Org 9426, a New Non-Depolarizing Neuromuscular Blocking Agent, in Patients Anaesthetized with Nitrous Oxide, Halothane, and Fentanyl. Canadian Journal of Anesthesia, 38, 430-435. http://dx.doi.org/10.1007/BF03007578

[19] Vermeyen, K.M., Hoffmann, V.L. and Saldien, V. (2003) Target Controlled Infusion of Rocuronium: Analysis of Effect Data to Select a Pharmacokinetic Model. British Journal of Anaesthesia, 90, 183-188. http://dx.doi.org/10.1093/bja/aeg043

[20] Hemmerling, T.M. and Donati, F. (2003) Neuromuscular Blockade at the Larynx, the Diaphragm and Corrugators Supercilii Muscle: A Review. Canadian Journal of Anesthesia, 50, 779-794. http://dx.doi.org/10.1007/BF03019373 